\title{
MODELO SIG PARA SELECÇÃO DE LOCAIS PARA ATERROS DE RESÍDUOS
}

\author{
C. N. $\operatorname{Costa}{ }^{1 *} ;$ A. $\operatorname{ALLEN}^{2}$; M. G. BRIto ${ }^{1}$; \\ P. S. Caetano ${ }^{1}$; V. Cummins ${ }^{3}$; J. Donnelly ${ }^{4}$; \\ S. Koukoulas ${ }^{3}$; V. O'Donnell ${ }^{3}$; C. Robalo ${ }^{5}$; D. Vendas ${ }^{1}$
}

\begin{abstract}
Resumo - A deposição em aterro constitui ainda o principal destino final dado aos resíduos produzidos na UE. A necessidade de garantir a protecção do ambiente e de conferir uma abordagem científica e mais transparente ao processo de selecção de locais para instalação de aterros constitui a motivação para o desenvolvimento do modelo de SIG apresentado neste trabalho.

O modelo teórico de SIG encontra-se estruturado em duas fases. Numa

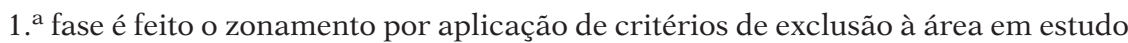
através da identificação de constrangimentos legais, sociais, logísticos e geológico/ /hidrogeológicos. Obtêm-se, deste modo, áreas residuais que, numa 2. ${ }^{a}$ fase, são sujeitas a ponderação dos parâmetros que intervêm na classificação em termos de aptidão para a instalação de aterros.
\end{abstract}

Palavras-chave : SIG, aterros de resíduos, ambiente, ordenamento do território.

Abstract - GIS MODEL FOR WASTE DISPOSAL SITES. Landfill is still the major method for waste disposal in the EU. The need to guarantee environmental protection and to follow a scientific and more transparent procedure of landfill site selection was the motivation to develop the GIS model presented in this paper. This work was developed under the auspices of an Interreg-funded EU project, by a team of Irish and Portuguese partners from University College Cork and Universidade Nova de Lisboa, and from local authorities in both countries, Cork County Council and Sesimbra Town Council.

* Carlos Nunes da Costa, Prof. Auxiliar. Centro de Investigação em Geociências Aplicadas da Universidade Nova de Lisboa. Quinta da Torre, 2829-516 Caparica. Tel/Fax: +351212948358. E-mail: cnc@fact.unl.pt

1 Centro de Investigação em Geociências Aplicadas da UNL, Portugal.

2 University College Cork, Eire.

3 Coastal Resources Centre/UCC, Eire.

4 Cork County Council, Eire.

5 Câmara Municipal de Sesimbra, Portugal. 
The theoretical GIS model consists of two steps. In the first step, data in vector structure and geo-processing techniques such as overlay and buffering are used to exclude areas unsuitable for landfill due to risk to the environment or human health. The exclusion factors considered in this step were grouped into anthropic and natural.

The remaining residual areas, considered suitable for the location of landfills, may have some land parcels more suitable than others, and are thus further examined in the second step of the GIS model. This step is an automated rasterbased process in which a series of parameters are used to classify each area in terms of high, moderate or low suitability for landfill. These parameters were also grouped into four different types: a) susceptibility to natural hazards; b) aquifer protection levels; c) land use; d) geo-environmental engineering. These parameters can also be weighted in order to provide different evaluation scenarios of the residual areas; extensions have been developed for ArcView GIS that will automatically calculate the weights for each category following inputs from the user.

Study sites in both countries were used to test and validate the model. This paper presents the results obtained from the application of the model to Sesimbra Council in Portugal.

The development of the landfill GIS model fulfils legislative and environmental obligations associated with site selection in a non-biased way, and the methodology aims at providing a clear and scientific rationale behind the choice of a site. Application of the model does not intend to substitute political decision but will provide decision makers (local authorities, waste management entities, etc.) with an important tool and a methodology that will aid their decision and enable them to conduct their own GIS landfill site selection screening process.

Key words : GIS, waste landfills, environment, land planning.

Resumé - Un modèle SIG POUR LE CHOIX DES SITES DESTINÉS AUX REMBlais D'ORDURES. L'accumulation des ordures en remblai continue à être la solution la plus souvent utilisée dans l'Union Européenne. La nécessaire protection de l'environnement et la recherche d'une procédure scientifique et transparente pour le choix des sites, expliquent la création du modèle SIG ici présenté. Il est l'œuvre conjointe de chercheurs de l'University College de Cork et de l'Universidade Nova de Lisbonne, en collaboration avec le Cork County Council et la mairie de Sesimbra, et le financement de l'Union Européenne.

Le modèle comprend deux phases. Sont d'abord exclus les secteurs qui ne conviennent pas pour des raisons légales, sociales, logistiques, géologiques ou hydrogéologiques. Les secteurs considérés comme aptes sont ensuite classés selon une série de paramètres: leur susceptibilité aux risques naturels, le niveau de protection de la nappe aquifère, le type d'occupation du sol, la technologie géoenvironnementale. Le poids de chaque paramètre est calculé automatiquement.

On a validé le modèle par l'étude de sites choisis en Irlande et au Portugal et l'exemple de la commune de Sesimbra est présenté.

L'application du modèle n'entend pas se substituer à la décision politique, mais fournir aux décideurs un outil qui facilite beaucoup leur choix.

Mots-clés : SIG, remblais d'ordures, environnement, aménagement du territoire. 


\section{INTRODUÇÃO}

Na União Europeia, a deposição controlada em aterro constitui ainda o principal destino final dos resíduos sólidos produzidos, quer sejam urbanos ou industriais, perigosos ou banais, e estima-se que continue a sê-lo nos tempos mais próximos. A premissa de que o revestimento de aterros é suficiente para a protecção do ambiente e a crença de que o projecto de engenharia poderá resolver situações geologicamente menos favoráveis tem relegado para segundo plano o processo de selecção de locais adequados para a instalação de aterros. No entanto, alguns trabalhos (SURmann et al., 1995) têm provado que os revestimentos utilizados em aterros podem sofrer deterioração considerável ao longo do tempo por acção do efeito corrosivo dos lixiviados produzidos. Por esta razão, é prudente procurar e desenvolver locais para aterro, que possuam características naturais que forneçam protecção secundária ("passiva») para o ambiente, na eventualidade de o revestimento do aterro não funcionar e, portanto, dar uma maior ênfase a todo o processo de selecção de locais.

Com o surgimento de novas tecnologias, nomeadamente de detecção remota e SIG, e consequente produção facilitada de mapas digitais, é possível desenvolver uma abordagem mais sistematizada ao problema da selecção dos melhores locais para instalação de aterros. Uma metodologia eficiente para a selecção de locais para aterro com as características naturais mais adequadas deverá, idealmente, combinar uma avaliação das condições geológicas, hidrogeológicas e geotécnicas locais com a aplicação de ferramentas SIG (ALLEN et al., 1997).

A motivação para o desenvolvimento de um modelo SIG com vista à selecção de locais para aterro reside numa combinação de factores, que incluem a necessidade de uma abordagem científica e não preconceituada na escolha dos locais, e a necessidade de contribuir para um aumento da confiança por parte do público numa base científica e transparente de todo o processo.

Neste trabalho, é apresentado um modelo SIG para apoio à selecção de locais para instalação de aterros, desenvolvido por uma equipa multidisciplinar (geólogos, engenheiros geólogos e civis, e especialistas em SIG), de parceiros irlandeses e portugueses oriundos de Universidades (University College Cork e Universidade Nova de Lisboa) e de autoridades locais dos dois países (Cork County Council e Câmara Municipal de Sesimbra). Este trabalho foi realizado no âmbito de um projecto internacional, financiado pelo Programa INTERREG IIC-Espaço Atlântico da UE, intitulado «The Development of a GIS Model for the Location of Landfill Sites in Ireland and Portugal».

O modelo desenvolvido visa identificar parcelas de território que, pelas suas características intrínsecas, revelem aptidão para a instalação de aterros de resíduos, independentemente do tipo de resíduos considerados (urbanos, industriais ou inertes). Deste modo, outros factores não intrínsecos aos locais, como os relacionados com acessibilidades, definição de fontes produtoras de resíduos, análises custo/distância e factores psico-sociais, poderão ser remetidas 
para outras fases do processo de selecção ou tratados com outros modelos (socio-ambientais de localização de infra-estruturas de resíduos; LoBER, 1995; MARQues et al., 2003). Assim, os parâmetros e critérios utilizados para o estabelecimento destas parcelas levam em consideração a identificação de um vasto conjunto de constrangimentos legais, sociais, ambientais e geológico/hidrogeológicos, que caracterizam a área de estudo. A todos estes factores de exclusão são aplicadas distâncias de protecção (buffers), com base ou em normas legais ou em recomendações técnicas constantes em bibliografia especializada. A possibilidade de aplicação do modelo teórico a situações de deposição em aterro de diferentes tipos de resíduos está prevista na medida em que é possível não só adequar os factores de exclusão a cada situação, como fazer variar as distâncias de protecção (dentro dos limites definidos). Da aplicação destes critérios de exclusão, resulta a definição de áreas consideradas como inaptas para a instalação de aterros - as áreas de exclusão - e de outras consideradas genericamente como aptas - as áreas residuais. Numa fase seguinte de aplicação do modelo, estas áreas são analisadas de modo a identificar diferentes graus de aptidão. O objectivo deste tipo de abordagem é reduzir significativamente a área seleccionada para estudos de pormenor em fases subsequentes do processo, podendo constituir-se uma carteira de sítios potenciais para instalação de aterros de resíduos que possam assim corresponder a solicitações que previsivelmente venham a ocorrer.

Toda a concepção deste modelo foi feita de modo a atribuir-lhe uma estrutura flexível de selecção de locais, que lhe confere uma capacidade transferível e transnacional podendo, tanto na UE como internacionalmente, servir como ferramenta de apoio à decisão a entidades como as autoridades locais, que estarão certamente envolvidas em processos de selecção de locais para aterro nos tempos mais próximos.

\section{O PROCESSO DE SELECÇÃO DE LOCAIS PARA ATERRO}

Qualquer procedimento desenvolvido para a selecção dos melhores locais para a instalação de aterros de resíduos deverá ter em atenção os seguintes objectivos:

a) Assegurar que o local seleccionado seja o mais adequado do ponto de vista ambiental, em termos de critérios técnicos que envolvam a saúde humana, a flora e fauna, o solo, a água e o ar, o clima e a paisagem;

b) Integrar o processo de selecção num programa mais abrangente de desenvolvimento regional, levando em consideração os factores económicos e legais inerentes;

c) Promover o consenso do público sobre a necessidade do aterro e a percepção de que todo o processo de selecção tomou em consideração, de um modo científico e equilibrado, todos os factores, nomeadamente os relacionados com outros interesses sectoriais (e.g. agricultura, indústria e turismo). 
Tomando em consideração os objectivos acima enunciados, e o modo como se poderia contribuir para os atingir, foi desenvolvido o modelo de SIG teórico para o apoio à selecção de locais para instalação de aterros de resíduos. Este modelo encontra-se estruturado em duas fases (fig. 1): numa $1 .^{\text {a }}$ fase é feito o zonamento da área em estudo por aplicação de critérios de exclusão estabelecidos a partir de um conjunto de factores definidos em dois tipos: os factores antrópicos e os factores naturais. No final desta fase a área de estudo encontra-se dividida em áreas de exclusão e em áreas que se definem como áreas residuais. Numa 2. ${ }^{a}$ fase de aplicação do modelo estas áreas residuais são sujeitas a avaliação através de parâmetros que intervêm na sua classificação em termos de graus de aptidão para a instalação de aterros. Estes parâmetros de avaliação estão igualmente agrupados em diferentes tipos: a) susceptibilidade a riscos naturais; b) níveis de vulnerabilidade de aquíferos; c) ocupação do solo e d) engenharia geoambiental.

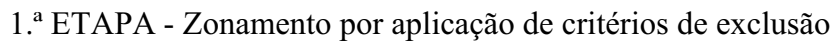

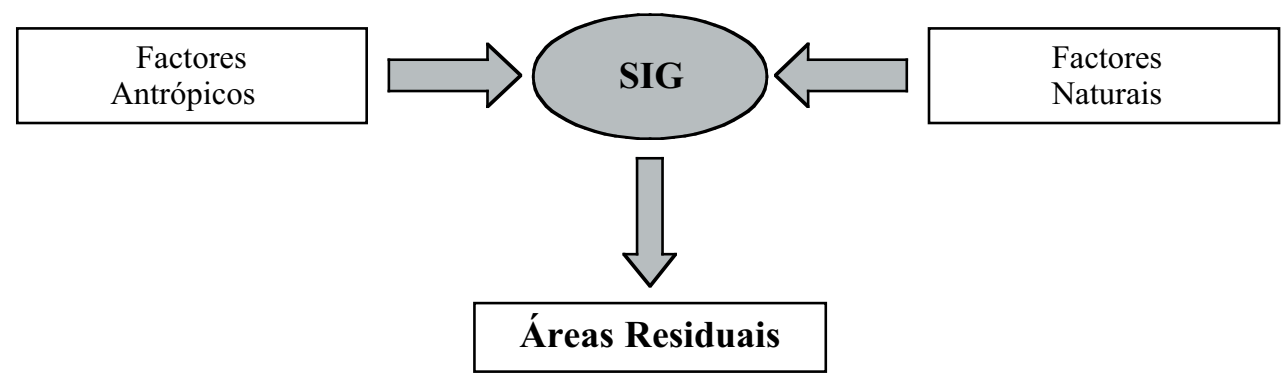

2. ETAPA - Ponderação de áreas residuais

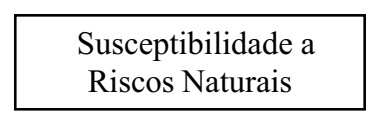

Níveis de Protecção de Aquíferos

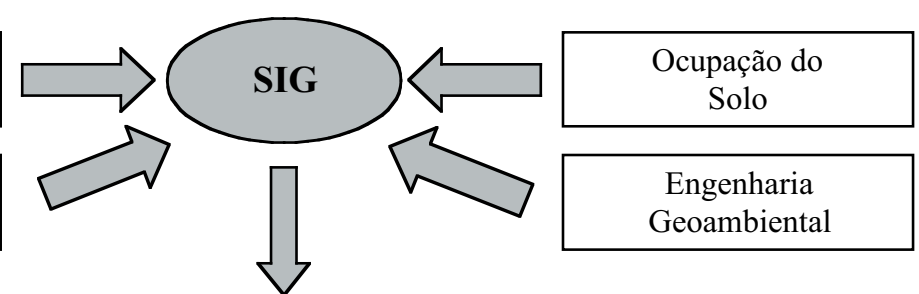

Sítios Potenciais

Fig. 1 - As duas fases de desenvolvimento do modelo SIG.

Fig. 1 - The two steps of the GIS model. 


\section{Aplicação de critérios de exclusão}

As áreas de exclusão correspondem a áreas onde, quer devido aos riscos para o ambiente quer para a saúde pública, um aterro não deverá ser instalado. Esta fase de aplicação do modelo não é automática e requer, por parte do utilizador, a captura, a introdução e a manipulação de dados. O processo de captura de dados visa obter em formato digital o maior número possível das variáveis necessárias; assim, quando inexistentes, é necessário proceder à sua digitalização. Nesta fase, os dados deverão encontrar-se em estrutura vectorial e, para criar as áreas de exclusão, são utilizadas técnicas de geo-processamento, tais como as de sobreposição, união e de aplicação de distâncias de protecção (buffering). No modelo desenvolvido são feitas recomendações sobre as distâncias de pro-

Quadro I - Factores de exclusão aplicados na $1 .^{\text {a }}$ fase do modelo SIG.

Table I-Exclusion factors for the $1^{\text {st }}$ step of the GIS model.

\begin{tabular}{|c|c|}
\hline 1 - Antrópicos & 2 - Naturais \\
\hline $\begin{array}{l}\text { A. Áreas construídas } \\
\text { A1. Áreas urbanas e industriais de alta tecno- } \\
\text { logia } \\
\text { A2. Áreas industriais: (perigosas, manufactu- } \\
\text { ração, alimentar/agrícola) } \\
\text { A3. Aeroportos (nacionais, locais, corredores } \\
\text { de aproximação) } \\
\text { A4. Estradas (principais, municipais) } \\
\text { A5. Linhas férreas } \\
\text { A6. Abastecimento de água: } \\
\text { 6.1. Reservatórios } \\
\text { 6.2. Poços, furos de captação } \\
\text { A7. Áreas Militares } \\
\text { A8. Edifícios públicos / Infra-estruturas (fora } \\
\text { de áreas urbanas/industriais; ex.: Hospi- } \\
\text { tais, Escolas, ETAR, Bombas de Gaso- } \\
\text { lina) } \\
\text { A9. Infra-estruturas lineares (condutas de } \\
\text { fluídos, condutas eléctricas, etc.) } \\
\text { B. Áreas classificadas } \\
\text { B1. Reservas naturais (REN, Rede Natura, } \\
\text { reservas cinegéticas, etc.) } \\
\text { B2. Reservas agrícolas e de caça } \\
\text { B3. Reservas arqueológicas e património } \\
\text { geológico } \\
\text { B4. Âreas mineiras (activas) } \\
\text { B5. Áreas de lazer / Outras } \\
\text { (espeleológicas, parques, etc.) }\end{array}$ & $\begin{array}{l}\text { B. Factores hidrológicos e geomorfológicos } \\
\text { B1. Rios (Permanentes/Temporários), Canais } \\
\text { B2. Planícies de inundação } \\
\text { B3. Lagos/Pântanos } \\
\text { B4. Linha de costa } \\
\text { B5. Declives }\end{array}$ \\
\hline
\end{tabular}


tecção que deverão ser utilizadas para cada factor de exclusão. Estas recomendações têm como base legislação vigente na UE sobre selecção de locais para aterro e uma exaustiva consulta bibliográfica (Allen et al., 2001; 2002).

No essencial, este processo de exclusão retira estas áreas de qualquer consideração futura no modelo, reduzindo significativamente a área que se mantém sob estudo. A lista de factores de exclusão, agrupados em Factores antrópicos e Factores naturais, encontra-se no Quadro I.

\section{Avaliação de áreas residuais}

Após aplicação dos critérios de exclusão da 1 . $^{\text {a }}$ fase, as áreas não excluídas são designadas de áreas residuais e, em função da sua não exclusão, são consideradas potencialmente aptas para a instalação de um aterro; no entanto, deve considerar-se que algumas destas áreas poderão ser mais aptas do que outras. Sendo assim, todas as áreas residuais deverão ser examinadas em função de um conjunto de parâmetros de avaliação no sentido de as poder hierarquizar em função da sua maior ou menor aptidão para a instalação de aterros. Os parâmetros de avaliação considerados no modelo SIG estão agrupados em quatro tipos (Quadro II).

Quadro II - Parâmetros de avaliação de áreas residuais.

Table II - Evaluation parameters of residual areas.

\begin{tabular}{|c|c|}
\hline Tipos & Parâmetros \\
\hline A - Susceptibilidade a riscos naturais & $\begin{array}{l}\text { 1. Movimentos de massa (deslizamentos, avalanches, } \\
\text { subsidência, solifluxão, etc.) } \\
\text { 2. Processos de erosão activa } \\
\text { 3. Actividade vulcânica } \\
\text { 4. Sismicidade } \\
\text { 5. Áreas susceptíveis a inundação }\end{array}$ \\
\hline B - Níveis de protecção de aquíferos & $\begin{array}{l}\text { 1. Importância local } \\
\text { 2. Vulnerabilidade } \\
\text { 3. Áreas de recarga }\end{array}$ \\
\hline C - Ocupação do solo & $\begin{array}{l}\text { 1. Ecológica (natural) } \\
\text { 2. Agrícola e silvícola } \\
\text { 3. Geológica }\end{array}$ \\
\hline D - Engenharia geoambiental & $\begin{array}{l}\text { 1. Barreira geológica natural (análise integrada de: } \\
\text { camadas/estrutura geológica/permeabilidade/espes- } \\
\text { sura/homogeneidade) } \\
\text { 2. Escavabilidade dos terrenos de fundação (facilidade } \\
\text { de escavação/ripabilidade) } \\
\text { 3. Capacidade de carga da fundação (processos de } \\
\text { ruptura, processos de assentamento) } \\
\text { 4. Estabilidade de taludes da fundação } \\
\text { 5. Potencial de regeneração do solo/custos de remediação }\end{array}$ \\
\hline
\end{tabular}


Nesta fase do modelo SIG, os dados introduzidos encontram-se em estrutura matricial e todos os parâmetros são categorizados com valores numéricos de acordo com o seu grau de susceptibilidade/adequabilidade utilizando-se o método (Delphi), baseado na avaliação pericial das características do território residual. Os parâmetros do tipo A, B e C são considerados como possuindo parâmetros desfavoráveis no que respeita à localização de um aterro; estes parâmetros são categorizados com valores negativos de acordo com o grau elevado, médio ou baixo de susceptibilidade à ocorrência de um risco natural (caso do tipo A), ou à susceptibilidade de ocorrência de danos ou perda de um recurso (casos dos tipos B e C). Os parâmetros do tipo D são considerados favoráveis à instalação de um aterro sendo categorizados com valores positivos, de acordo com o seu grau elevado, médio ou baixo de adequabilidade para instalação de um aterro. À não aplicabilidade de um parâmetro é atribuído um valor nulo (zero).

Para além da atribuição de um grau de susceptibilidade ou adequabilidade, é possível atribuir também um peso a cada parâmetro de avaliação de áreas residuais. Deste modo, o processo permite a formulação de diferentes cenários consoante a importância relativa atribuída a cada parâmetro. Esta atribuição de pesos aos diferentes parâmetros poderá ser efectuada tendo por base diferentes sensibilidades técnico-científicas, ou outras, e será necessariamente diferente consoante a entidade que os atribui (e.g. será possível favorecer as características geotécnicas dos diferentes locais atribuindo um maior peso aos parâmetros

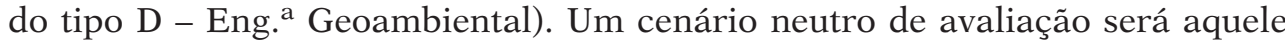
que é produzido por uma atribuição de pesos idênticos a cada parâmetro.

O modelo SIG permite assim ao utilizador avaliar as áreas residuais com base num conjunto de critérios e objectivos, múltiplos e opostos. O Método de Análise Hierárquica (Analytic Hierarchy Process), proposto por T. SAATY (1980), é uma das abordagens possíveis para a realização desta avaliação multi-critério e foi adoptado no modelo. Neste método, faz-se uma comparação par-a-par dos parâmetros de avaliação produzindo, no final, uma escala hierárquica de ponderação para cada um dos parâmetros considerados. O método de Saaty tem sido largamente utilizado e é considerado como um dos métodos mais adequados para avaliação e escalonamento hierárquico de alternativas em problemas multi-critério de tomada de decisão (Triantaphyllou, 1985). No entanto, o modelo foi concebido de modo a permitir a introdução da ponderação dos parâmetros por outros métodos. No desenvolvimento do modelo SIG, foram criadas extensões para ArcView GIS, que calculam automaticamente os pesos dados a cada parâmetro a partir dos dados introduzidos pelo utilizador, independentemente dos métodos usados.

\section{Avaliação total das áreas residuais e identificação de sítios potenciais}

Após a categorização e atribuição de um factor de ponderação a cada parâmetro de avaliação, a avaliação total (at) das áreas residuais é calculada de acordo com a seguinte expressão: 


$$
a t=\sum_{i=1}^{p a r} C_{i} \times \alpha_{i}
$$

onde, $C i=$ valor do parâmetro $i$;

$\alpha i=$ peso atribuído ao parâmetro $i$;

par $=$ número de parâmetros.

A gama dos valores obtidos de at indica a maior ou menor aptidão dos locais para a instalação de um aterro. Consoante o tipo de distribuição destes valores, a sua divisão em classes poderá ser feita de diversos modos, por exemplo: classes definidas a partir do valor médio e da subtracção e soma de múltiplos do desvio padrão (no caso de uma distribuição gaussiana); ou classes de igual amplitude (no caso de uma distribuição uniforme ou bimodal). $\mathrm{O}$ resultado final desta divisão, quando feita em três classes, produz uma hierarquização das áreas residuais em termos da classificação da sua maior, média ou menor aptidão para a instalação de um aterro.

Na sequência da 2. ${ }^{a}$ fase, as áreas mais aptas deverão continuar a ser analisadas utilizando outras técnicas como estudos de impactes na paisagem, análises custo/distância, acessibilidades e de condições meteorológicas. Da lista ordenada de aptidão é possível identificar um conjunto dos melhores locais definindo sítios potenciais que deverão, posteriormente, ser sujeitos a avaliação técnica detalhada de campo, em particular com estudos geotécnicos e hidrogeológicos de pormenor.

\section{APLICAÇÃO DO MODELO TEÓRICO}

Para além da concepção e desenvolvimento de um modelo teórico de SIG para apoio à selecção de locais para instalação de aterros de resíduos, dos objectivos do projecto INTERREG constavam testes de aplicação do modelo. Nesse sentido, foram definidas duas áreas de estudo: a região sul de Cork County, na Irlanda, e o concelho de Sesimbra, em Portugal. Em ambos os casos, a aplicação do modelo não tinha como intenção responder à necessidade de resolução de problemas relacionados com a gestão de resíduos, mas funcionar apenas como teste de validação do modelo. Neste trabalho apresentam-se os resultados obtidos da aplicação do modelo à área teste de Sesimbra.

\section{Caracterização da área teste de Sesimbra}

O concelho de Sesimbra situa-se no extremo SW da Península de Setúbal, a cerca de $20 \mathrm{~km}$ de Lisboa, limitado a sul e oeste pelo litoral Atlântico, a norte pelos concelhos de Almada e Seixal e a este pelo de Setúbal (fig. 2). Ocupa uma área de $206 \mathrm{~km}^{2}$ com uma população de cerca de 38000 habitantes, que na época alta de Verão pode atingir os 100000 . A região de Sesimbra é essencialmente 


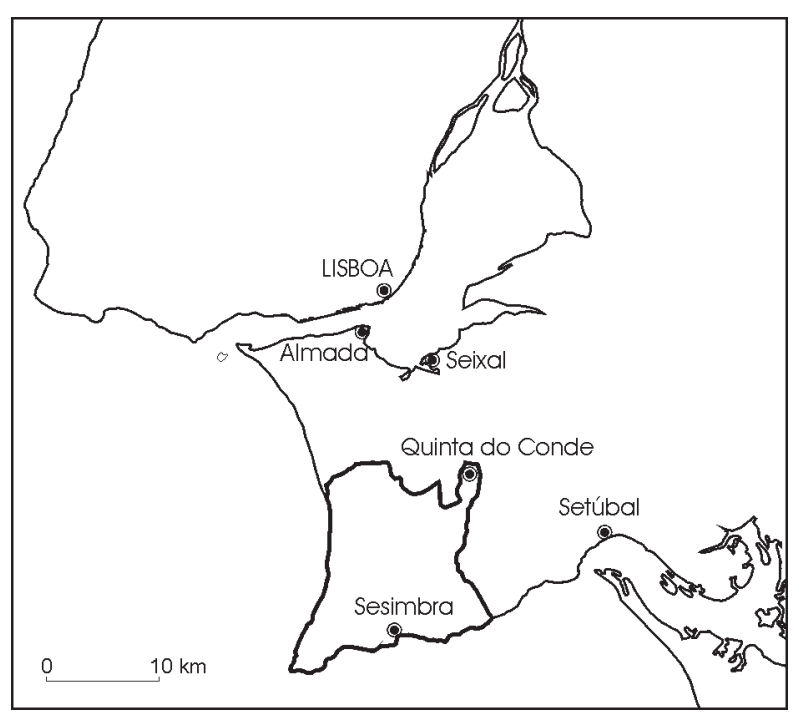

Fig. 2 - Localização do concelho de Sesimbra.

Fig. 2 - Location of the Sesimbra municipality study area.

um destino turístico com actividade piscatória, algumas áreas rurais e de floresta. Existem dois centros urbanos principais - a Vila de Sesimbra, com cerca de 6000 habitantes, e a da Quinta do Conde, com cerca de 16000. Topograficamente, a área sul do concelho é relativamente acidentada, integrada no extremo ocidental da cadeia da Arrábida, com litoral rochoso e escarpado; para norte, encontram-se a Ribeira da Apostiça e a Lagoa de Albufeira, sendo a região relativamente plana.

\section{Zonamento da área teste por aplicação de critérios de exclusão}

A captura de dados para aplicação da $1 .{ }^{\mathrm{a}}$ fase do modelo SIG revelou-se um trabalho muito moroso. Com efeito, muito pouca informação estava disponível em formato digital, tendo sido necessário proceder à vectorização da grande maioria dos parâmetros considerados. As fontes de informação utilizadas nesta fase foram essencialmente cartas topográficas do Instituto Geográfico de Exército, plantas de ordenamento e condicionantes do Plano Director Municipal, Rede Natura, cartas geológicas e outras cartas de factores (e.g. arqueológica e hidrogeológica). Nesta fase, a escala de trabalho é essencialmente condicionada pelas escalas disponíveis nas fontes de informação; na sua grande maioria, as fontes utilizadas nesta aplicação apresentam escalas de 1/25 000 e 1/50 000.

Após atribuição de distâncias de protecção e união da informação, criou-se um mapa de exclusão para todo o concelho; ao complementar destas áreas de exclusão, correspondem as áreas residuais a avaliar na $2 .^{\mathrm{a}}$ fase de aplicação do 
modelo. Com o objectivo de eliminar áreas residuais de dimensão insuficiente para instalação de um aterro foi determinado que todas as áreas residuais inferiores a 10ha fossem também excluídas (no modelo este valor pode ser alterado consoante a dimensão do aterro a projectar). Após esta operação foi produzido um mapa final de áreas residuais (fig. 3), que totalizam aproximadamente 15\% da área total do concelho, significando uma redução assinalável da área a considerar nas fases seguintes do estudo.

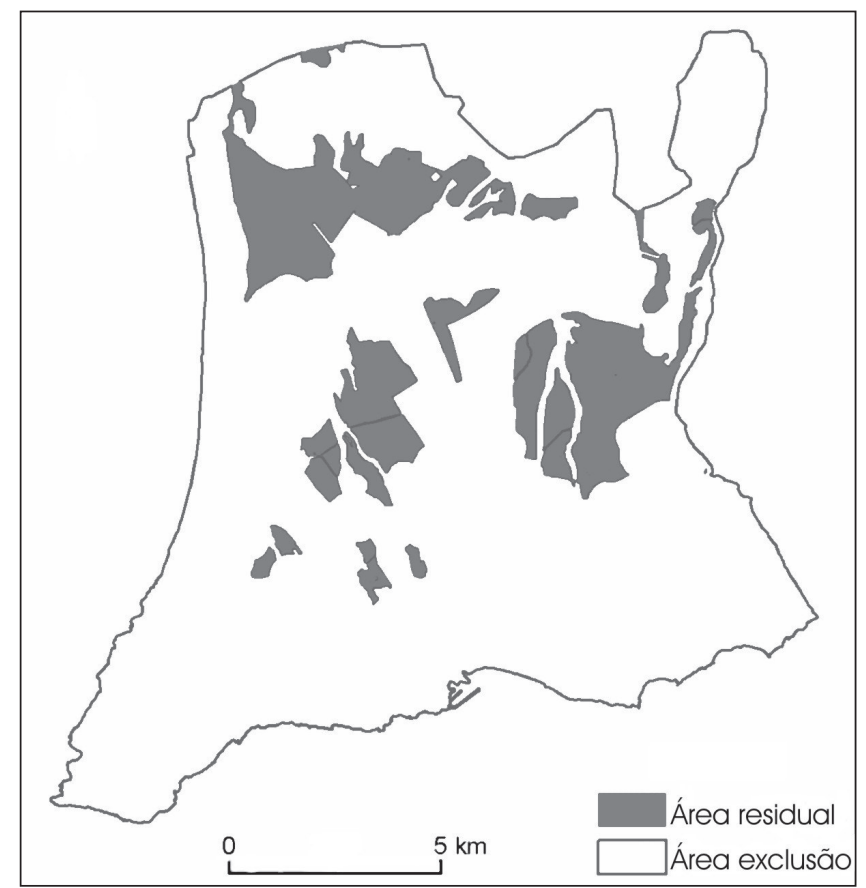

Fig. 3 - Distribuição de áreas de exclusão vs. áreas residuais no concelho de Sesimbra.

Fig. 3 - Exclusion areas vs. residual areas in the Sesimbra municipality.

\section{Ponderação de áreas residuais na área teste}

A aplicação da $2 .^{a}$ fase do modelo SIG requer igualmente a captura de um vasto conjunto de dados, a maioria dos quais, mais uma vez, não se encontram em formato digital. Devido à inexistência de elementos referentes a alguns dos parâmetros de avaliação, até mesmo em formato analógico, estes foram derivados de outras fontes por avaliação pericial (e.g. parâmetros do tipo D Engenharia geoambiental, a partir de cartas geológicas). Por outro lado, alguns parâmetros não têm aplicação na área teste do concelho de Sesimbra (e.g. «actividade vulcânica») e outros não foram determinados por falta de informação (e.g. «movimentos de massa»). Nas figuras 4, 5, 6 e 7 são apresentados alguns 


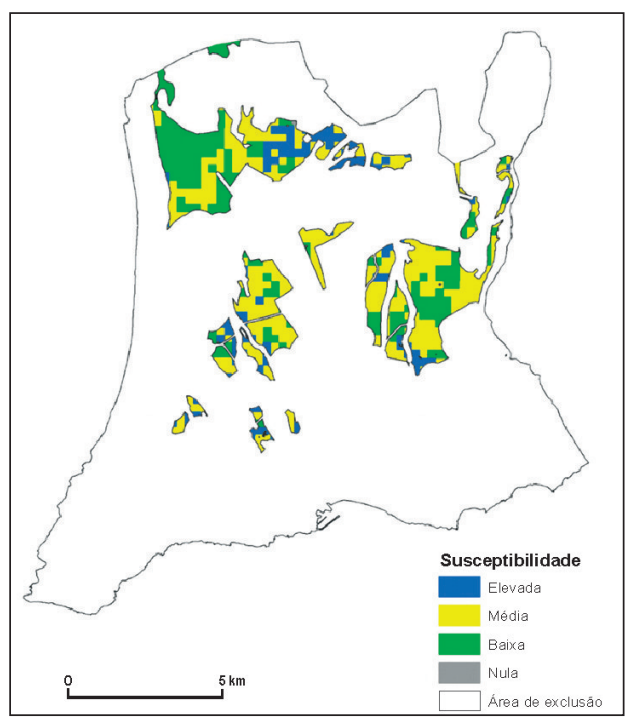

Fig. 4 - Categorização da susceptibilidade à ocorrência de processos de erosão activa.

Fig. 4 - Classification of active erosion processes susceptibility.

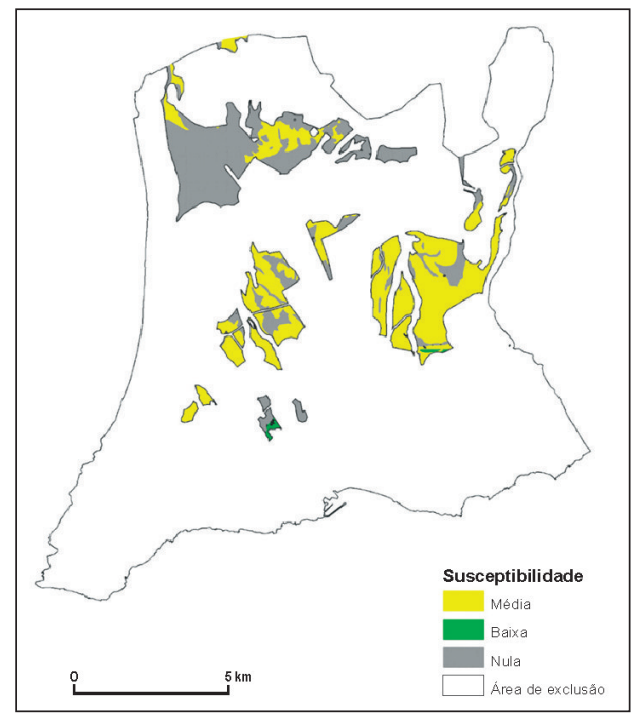

Fig. 6 - Categorização da susceptibilidade ao dano, ou perda, para os recursos geológicos.

Fig. - Classification of damage or loss to geological resources susceptibility.

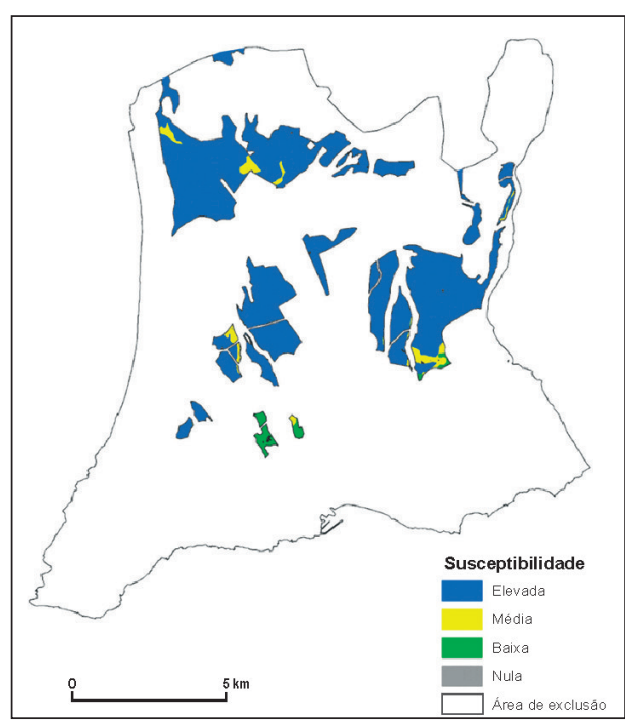

Fig. 5 - Categorização da susceptibilidade ao dano, ou perda, para os aquíferos em função da sua vulnerabilidade.

Fig. 5 - Classification of aquifer damage susceptibility due to vulnerability.

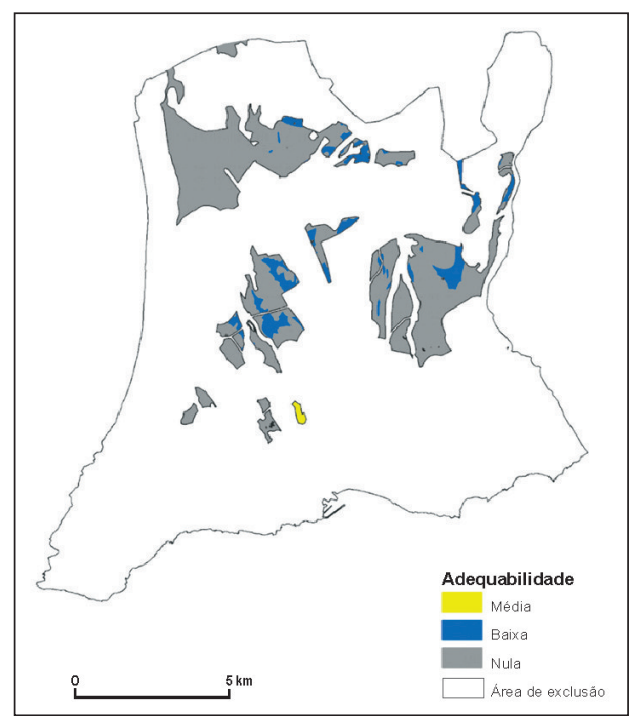

Fig. 7 - Categorização da adequabilidade da área em termos de existência de barreira geológica natural.

Fig. 7 - Classification of residual area suitability in terms of natural geological barrier. 
exemplos da categorização de parâmetros de avaliação das áreas residuais do concelho de Sesimbra em termos da sua elevada, média ou baixa susceptibilidade/adequabilidade. É de salientar que, nesta fase, a avaliação pericial destes parâmetros irá apenas incidir numa parcela do território correspondente a cerca de $15 \%$ do concelho, o que significa uma redução substancial do esforço implicado nesta tarefa.

Como foi referido, a aplicação da $2 .^{\text {a }}$ fase do modelo SIG requer toda a informação em estrutura matricial; em alguns casos, a rasterização da informação foi feita somente após a sua categorização em estrutura vectorial. $\mathrm{Na}$ figura 8 é apresentado um mapa final de avaliação e respectiva hierarquização de áreas residuais em termos da sua elevada, média ou baixa aptidão para instalação de aterros de resíduos. No exemplo desta figura, o cenário apresentado corresponde à atribuição de um peso igual a todos os parâmetros. A projecção de outro cenário diferente de hierarquização poderia ser obtida se fossem atribuídos pesos diferentes a cada parâmetro consoante a avaliação da sua maior ou menor importância.

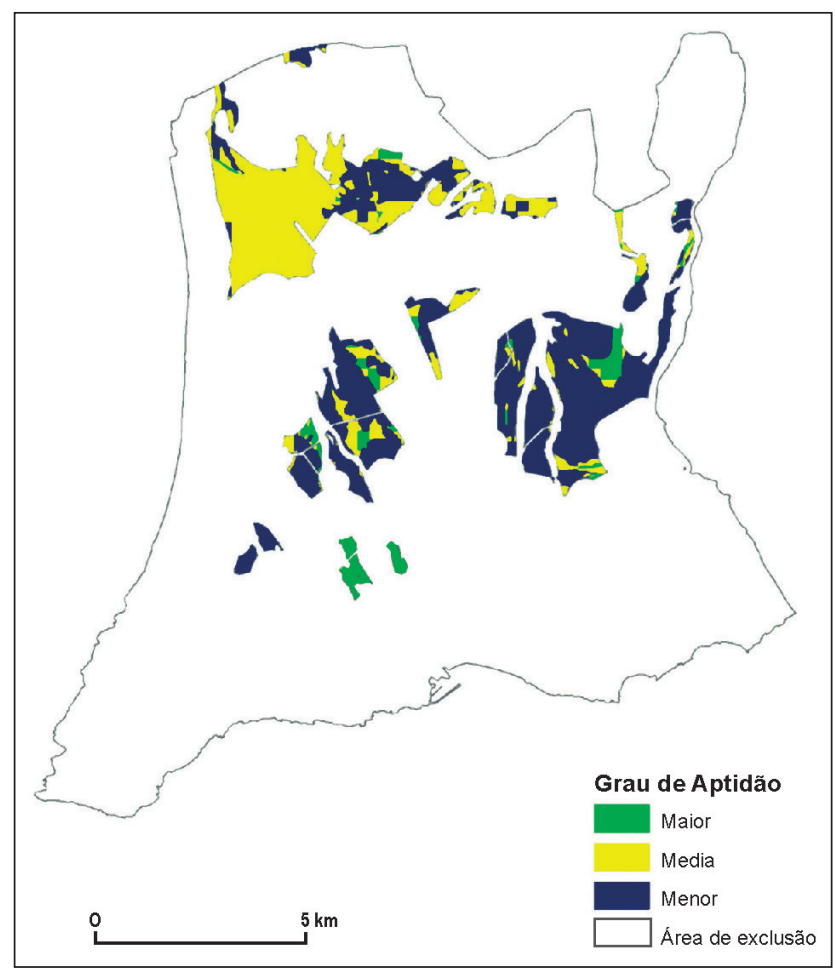

Fig. 8 - Avaliação total e hierarquização de áreas residuais em termos da sua maior, média ou menor aptidão para instalação de aterros.

Fig. 8 - Final evaluation and classification of residual areas in terms of high, medium and low suitability for landfill location. 


\section{CONSIDERAÇÕES FINAIS}

Em geral, a opinião pública opõe-se fortemente a processos de instalação de aterros de resíduos, em particular na fase de escolha da sua localização. Só um procedimento aberto e transparente na selecção de locais, com consulta e participação do público em todas as fases do processo, poderá minimizar ou inverter esta tendência. $\mathrm{O}$ envolvimento das populações locais deverá ser feito, num processo de selecção deste tipo, logo desde o seu início, através da disseminação de informação, consultas públicas e reuniões. Só assim será possível contrariar o chamado síndroma $N I M B Y$ e obter o apoio das populações aos procedimentos seguidos na selecção e consequente aceitação do resultado final.

A utilização de um modelo SIG como parte do processo de escolha de um local para a instalação de um aterro pode contribuir para tornar todo o processo mais racional. $\mathrm{O}$ modelo apresentado neste trabalho cumpre as obrigações legislativas e ambientais, procurando fornecer um fundamento científico no apoio à decisão de escolha do melhor local. A inclusão de métodos de avaliação multicritério contribui também para reduzir a subjectividade do exercício.

A aplicação do modelo teórico à área teste do concelho de Sesimbra permitiu, por um lado, verificar a validade do modelo, e, por outro, evidenciar a dificuldade em obter dados geo-referenciados em quantidade e de boa qualidade. Face a esta situação, sugere-se que um forte investimento seja feito, nomeadamente pelas autoridades locais, na obtenção e criação de bases de dados geo-referenciados, que permitam levar a cabo aplicações SIG do tipo do apresentado neste trabalho.

Do ponto de vista técnico, a abordagem faseada e em ambiente SIG da procura dos melhores locais para instalação de aterros aponta para uma maior rendibilidade dos estudos a efectuar, na medida em que, ao reduzir substancialmente o território a analisar através da definição de áreas de exclusão, se poderá direccionar essa tarefa para um conjunto relativamente reduzido e já pré-seleccionado de locais. Esta vantagem ficou demonstrada através da aplicação do modelo ao concelho de Sesimbra, onde a área residual total, resultante da aplicação de critérios de exclusão, e avaliada na $2{ }^{a}$ fase, correspondeu somente a cerca de $15 \%$ do território concelhio.

Este modelo SIG não pretende substituir a decisão política, antes fornecendo às entidades decisoras (e.g. Autarquias, Associações de Municípios, entidades gestoras de resíduos, etc.) uma ferramenta e uma metodologia importante de apoio a processos de selecção de locais para instalação de aterros.

\section{AGRADECIMENTOS}

Os autores agradecem o apoio financeiro do Programa INTERREG IIC - Espaço Atlântico ao desenvolvimento do projecto Ref. ${ }^{a}$ EA-B1IRE-n. ${ }^{\circ} 2.15$, no âmbito do qual foi desenvolvido este trabalho. 


\section{BIBLIOGRAFIA}

Allen, A. R.; Dillon, A. M. e O’Brien, M. (1997) - Approaches to landfill site selection in Ireland. In Marinos, P. G.; Koukis, G. C.; Tsiambaos, G. C. e Stournaras, G. C. (eds.) - Engineering Geology and the Environment. Balkema, Rotterdam: 1569-1574.

Allen, A. R.; Brito, M. G.; Caetano, P. S.; Costa, C. N.; Cummins, V.; Donnelly, J.; Fernandes, C.; Koukoulas, S.; O’Donnell, V.; Robalo, C. e Vendas, D. (2001) - The development of a GIS model for the location of landfill sites in Ireland and Portugal. Relatório Final. Projecto EA-B1IREn. 2.15 (193 p.) (não publicado).

Allen, A. R.; Brito, M. G.; Caetano, P. S.; Costa, C. N.; Cummins, V.; Donnelly, J.; Fernandes, C.; Koukoulas, S.; O’Donnell, V.; Robalo, C. e Vendas, D. (2002) - Procedure for the location of landfill sites using a GIS Model. In van Rooy, J. L. e Jermy, C. A. (eds.) - Engineering Geology for Developing Countries. Proceed. 9th Congress International Association of Engineering Geology and the Environment. Durban, South Africa: 2704-2713.

Lober, D. J. (1995) - Resolving the siting impasse - modelling social and environmental locational criteria with a geographic information system. Journal of the American Planning Association, vol. 61, n. ${ }^{\circ}$ 4, Autumn. American Planning Association, IL, Chicago: 482-495.

Marques, M. J.; Martinho, M. G.; Vasconcelos, L.; Maia, J.; Machado, F. e Painho, M. (2003) - Desenvolvimento de um modelo integrado de pré-localização de aterros de RSU, Parte 3 - Integração da componente social. VII Congresso Nacional de Engenharia do Ambiente. APEA, Lisboa.

SAATY, T. L. (1980) - The analytic hierarchy process. McGraw Hill, New York, 287 p.

Surmann, R.; Pierson, P. e Cottour, F. (1995) - Geomembrane liner performance and long-term durability. In Christensen, T. H.; Cossu, R. e Stegmann, R. (eds.) - Proceedings Sardinia 91, Third International Landfill Symposium, vol. 2. CISA Publisher: 405-414.

TriantaPHyLlou, E. (1985) - Evaluation of alternatives in single and multidimensional decision-making problems. MSc Thesis. The Pennsylvania State University (não publicado). 